\title{
Measurement of myocardial blood flow by cardiovascular magnetic resonance perfusion: comparison of distributed parameter and Fermi models with single and dual bolus
}

Giorgos Papanastasiou ${ }^{1,2 *}$, Michelle C Williams², Lucy E Kershaw³, Marc R Dweck², Shirjel Alam², Saeed Mirsadraee', Martin Connell', Calum Gray ${ }^{1}$, Tom MacGillivray ${ }^{1}$, David E Newby ${ }^{1,2}$ and Scott IK Semple $e^{1,2}$

\begin{abstract}
Background: Mathematical modeling of cardiovascular magnetic resonance perfusion data allows absolute quantification of myocardial blood flow. Saturation of left ventricle signal during standard contrast administration can compromise the input function used when applying these models. This saturation effect is evident during application of standard Fermi models in single bolus perfusion data. Dual bolus injection protocols have been suggested to eliminate saturation but are much less practical in the clinical setting. The distributed parameter model can also be used for absolute quantification but has not been applied in patients with coronary artery disease. We assessed whether distributed parameter modeling might be less dependent on arterial input function saturation than Fermi modeling in healthy volunteers. We validated the accuracy of each model in detecting reduced myocardial blood flow in stenotic vessels versus gold-standard invasive methods.
\end{abstract}

Methods: Eight healthy subjects were scanned using a dual bolus cardiac perfusion protocol at 3T. We performed both single and dual bolus analysis of these data using the distributed parameter and Fermi models. For the dual bolus analysis, a scaled pre-bolus arterial input function was used. In single bolus analysis, the arterial input function was extracted from the main bolus. We also performed analysis using both models of single bolus data obtained from five patients with coronary artery disease and findings were compared against independent invasive coronary angiography and fractional flow reserve. Statistical significance was defined as two-sided $P$ value $<0.05$.

Results: Fermi models overestimated myocardial blood flow in healthy volunteers due to arterial input function saturation in single bolus analysis compared to dual bolus analysis $(P<0.05)$. No difference was observed in these volunteers when applying distributed parameter-myocardial blood flow between single and dual bolus analysis. In patients, distributed parameter modeling was able to detect reduced myocardial blood flow at stress $(<2.5 \mathrm{~mL} / \mathrm{min} / \mathrm{mL}$ of tissue) in all 12 stenotic vessels compared to only 9 for Fermi modeling.

Conclusions: Comparison of single bolus versus dual bolus values suggests that distributed parameter modeling is less dependent on arterial input function saturation than Fermi modeling. Distributed parameter modeling showed excellent accuracy in detecting reduced myocardial blood flow in all stenotic vessels.

Keywords: Cardiovascular magnetic resonance, Myocardial blood flow, Fermi modeling, Distributed parameter modeling, Fractional flow reserve, Invasive coronary angiography

\footnotetext{
* Correspondence: G.Papanastasiou@ed.ac.uk

${ }^{1}$ Clinical Research Imaging Centre, University of Edinburgh „E Edinburgh, UK

${ }^{2}$ Centre for Cardiovascular Science, University of Edinburgh, Edinburgh, UK

Full list of author information is available at the end of the article
} 


\section{Background}

Mathematical modeling of cardiovascular magnetic resonance perfusion (CMR) has the potential to allow quantitative assessment of myocardial blood flow [1,2]. Establishing absolute quantification of blood flow could have clinical benefits since it may lead to an improvement in the diagnosis and prognostication of patients with coronary artery disease [3-6].

Myocardial blood flow quantification using modeldependent analysis is based on fitting the convolution of a model with the arterial input function to the tissue contrast agent concentration-time curve. The model describes the passage of a contrast agent through the myocardium whilst the arterial input function is the observed contrast agent concentration-time curve derived from the blood pool [3]. Fermi deconvolution modeling is a popular approach used to estimate myocardial blood flow during the first-pass of gadolinium-based extracellular contrast agents (CA) $[3,7,8]$. It is an empirical-mathematical model, which is convolved with the first-pass of the arterial input function [3]. The distributed parameter model assumes that the extravascular-extracellular space exchanges CA with nearby regions in the intravascular space, restricting axial transport of CA inside the extravascular-extracellular space [4]. In addition to myocardial blood flow, this model can also be used to calculate other microvascular characteristics including intravascular space, extravascular-extracellular space, permeability surface area product, extraction fraction and volume of distribution [9].

The high concentration of CA during bolus passage leads to signal saturation and causes concentration underestimation in the left ventricular cavity [10] (which is used to generate an arterial input function for model deconvolution analysis). This can degrade the accuracy and reproducibility of myocardial blood flow quantification using Fermi modeling, leading to systematic myocardial blood flow overestimation [3]. The dual bolus acquisition technique can eliminate signal saturation allowing more reliable quantification of myocardial blood flow in first-pass magnetic resonance perfusion imaging. In the dual bolus technique, an initial injection of dilute $\mathrm{CA}$ is used to acquire a non-saturated arterial input function before the main CA bolus. This is commonly referred to as the "pre-bolus" $[11,12]$. However, compared to single bolus protocols [1-3,7-9], dual bolus imaging protocols are characterized by increased complexity both in image acquisition and data analysis [3,10-12].

In the present study, we compared single and dual bolus estimates of myocardial blood flow in healthy volunteers using both distributed parameter and Fermi models. We also assessed whether these models can reliably detect areas with reduced myocardial blood flow compared to a clinical gold standard of invasive coronary angiography and fractional flow reserve in patients with coronary artery disease.

\section{Methods}

\section{Study population}

Eight healthy volunteers with no previous history of cardiovascular or renal disease, diabetes mellitus, asthma or any other clinically significant illness and five patients with suspected coronary artery disease were recruited into the study. Exclusion criteria included severely compromised renal function (estimated glomerular filtration rate $<30 \mathrm{~mL} / \mathrm{min}$ ) and contraindications to magnetic resonance imaging. The study was performed with the approval of the local research ethics committee, in accordance with the Declaration of Helsinki and with the written informed consent of all subjects. Prior to CMR perfusion, all subjects were asked to refrain from caffeine for 12 hours.

\section{Image acquisition}

All data were acquired using a 3T Verio system (Siemens AG, Healthcare Sector, Erlangen, Germany). Standard cardiac imaging planes and a short axis stack of left ventricular cine data were acquired using routine steady state free precession (TrueFISP) acquisitions. Native $\mathrm{T}_{1}$ relaxation rates (i.e. in the absence of $C A$ ) were calculated using the modified Look-Locker inversion (MOLLI) recovery technique [13]. Stress imaging was performed by intravenous infusion of $140 \mu \mathrm{g} / \mathrm{kg} /$ min of adenosine (Adenoscan, Sanofi Aventis). Fifty dynamic perfusion images were obtained at diastole across three short-axis view slices: basal, mid-ventricular and apical slices according to the standard 16-segment heart model [14]. Perfusion images were acquired using a turbo-fast low angle shot (FLASH) saturation recovery prepared single-shot gradient echo pulse sequence (repetition time/ echo time $2.20 \mathrm{~ms} / 1.07 \mathrm{~ms}$, flip angle $12^{\circ}$, slice thickness $8 \mathrm{~mm}$, preparation pulse delay (PD) to central line of k-space $100 \mathrm{~ms}$, matrix size $192 \times 108$ and FoV $330 \mathrm{~mm} \times 440 \mathrm{~mm}$ ). With the application of GRAPPA (accelerator factor of 3) and partial Fourier acquisition of 0.75 , each dynamic frame consisted of 48-phase encoded lines. All CMR perfusion data were acquired using electrocardiogram-gating.

\section{Contrast agent bolus administration}

In single bolus imaging, $0.05 \mathrm{mmol} / \mathrm{kg}$ of CA (Gadovist, Bayer Healthcare) was injected intravenously after $4 \mathrm{~min}$ of adenosine infusion, followed by $20 \mathrm{~mL}$ of $0.9 \%$ saline (Medrad Spectris Solaris, Medrad, USA) at $4 \mathrm{~mL} / \mathrm{s}$ [3]. All patients with coronary artery disease were imaged using the single bolus protocol. 
In the healthy volunteer cohort, an additional prebolus infusion was administered to allow dual bolus modeling to be applied without the risk of signal saturation in the arterial input function. In this dual bolus protocol, the two boluses were injected in a predetermined concentration ratio (pre-bolus:main bolus, $1: 5)$ with the pre-bolus diluted using $0.9 \%$ saline. After $3.5 \mathrm{~min}$ of adenosine infusion, the pre-bolus of 0.006 $\mathrm{mmol} / \mathrm{kg} \mathrm{CA}$ was injected and adenosine was continued until the main bolus of $0.03 \mathrm{mmol} / \mathrm{kg}$ had also been administered. The pre-bolus allows determination of the arterial input function whilst the main bolus allows measurement of myocardial CA concentration curves [15]. To allow clearance of residual CA, the rest perfusion imaging was performed 15 min after the adenosine-stress scan with the same acquisition protocol in all subjects $[3,7,8,15]$.

\section{Invasive coronary angiography and fractional flow reserve}

All five patients underwent invasive coronary angiography at the Royal Infirmary of Edinburgh. Fractional flow reserve was assessed for major epicardial vessels and defined as the ratio between distal coronary pressure and aortic pressure measured simultaneously at maximal adenosine-induced (intravenous $140 \mu \mathrm{g} / \mathrm{kg} / \mathrm{min}$ ) hyperaemia [16,17]. Haemodynamically significant coronary artery disease was defined as luminal stenosis $\geq 70 \%$ on invasive coronary angiography, or fractional flow reserve $<0.80$ and luminal stenosis $\geq 50 \%$. Outcomes from the three main coronary vessels were classified into 3 groups: Group 1, no or minor coronary artery disease with luminal stenosis $<50 \%$; Group 2, non-obstructive coronary artery disease with luminal stenosis $\geq 50 \%$ and fractional flow reserve $>0.80$; and Group 3, obstructive coronary artery disease with luminal stenosis of $\geq 70 \%$ alone, or luminal stenosis $\geq 50 \%$ and fractional flow reserve $\leq 0.80[16,17]$.

\section{Cardiac contouring}

Endocardial and epicardial contours were manually defined on the short axis magnetic resonance perfusion imaging data using dedicated cardiac image analysis software (QMass, Medis, The Netherlands) to generate a standardised 16-segment model of the heart [14]. Myocardial blood flow analysis was performed per myocardial segment. The signal intensity of the arterial input function was extracted from the left ventricular cavity excluding papillary muscles using customised in-house software created in Matlab (MathWorks Inc., Natick, MA) [18].

All arterial input function curves were extracted from the basal slice [3,7]. In single bolus analysis, the arterial input function was extracted from the standard (main bolus) CA dose component. For the dual bolus analysis in healthy subjects, the pre-bolus arterial input function was scaled and used for deconvolution analysis $[3,11,15]$.

\section{Image processing}

To correct for signal saturation, myocardial and arterial input function signal intensity-time curves were converted to CA concentration-time curves using the method of Larsson et al [19], as described previously $[3,7-9,11,12,19,20]$. This method is based on the assumption that in a region of interest, the longitudinal relaxation rate $R_{1}\left(1 / T_{1}\right)$ changes linearly as a function of contrast agent concentration influx $\mathrm{c}(\mathrm{t})$ at time $\mathrm{t}$ multiplied by its relaxivity $r_{1}$ according to the following equation:

$$
\frac{1}{\mathrm{~T}_{1}(\mathrm{t})}-\frac{1}{\mathrm{~T}_{1}(0)}=r 1 \cdot c(t)
$$

where $T_{1}(0)$ is the native longitudinal relaxation rate and $\mathrm{T}_{1}(\mathrm{t})$ is the longitudinal relaxation rate at time $\mathrm{t}$ of contrast enhancement. By substituting $\Delta R_{1} \frac{1}{\mathrm{~T}_{1}(\mathrm{t})}-\frac{1}{\mathrm{~T}_{1}(0)}$, equation (1) can be re-written as:

$$
c(t)=\frac{\Delta R_{1}}{r_{1}}
$$

In the above set of equations, $\mathrm{T}_{1}(\mathrm{t})$ is unknown and can be calculated by adapting the MR signal equation for the saturation recovery prepared single-shot FLASH sequence as follows $[3,7,19]$ :

$$
S I=\psi \cdot\left[\left(1-e^{-P D \cdot R_{1}}\right) \cdot a^{n-1}+b \cdot \frac{1-a}{1-a}\right]
$$

where SI is the signal intensity, $\Psi$ is a calibration constant dependent on receiver gain, instrumental conditions, proton density and $\alpha$. PD is the pre-pulse delay which is the time between saturation pulse and the central line of k-space, $a=\cos (\alpha) \cdot e^{-T R \cdot R_{1}}$ and $b=1-e^{-T R \cdot R_{1}}$. TR is the time interval between repetitive $\alpha$-radiofrequency pulses. $\Psi$ is assumed to be constant throughout the dynamic perfusion image acquisition [19] and was initially calculated from equation (3) by using native $T_{1}$ and signal intensities derived from a region of interest (i.e. myocardial segment, arterial input function) in the absence of CA. $T_{1}(t)$ at time $t$ of contrast enhancement was then calculated from equation (3) using $\Psi$ and signal intensity values extracted from the same region of interest in each of the dynamic perfusion images. CA concentration-time curves were then calculated using equation (2). 


\section{Model equations}

The model equations used for data fitting are summarized in Table 1. These equations represent the tissue impulse response $\mathrm{R}(\mathrm{t})$ the shape of which is determined by the fitted parameters [3]. To quantify myocardial blood flow and other parameters generated by the Fermi and distributed parameter models, we used modelconstrained deconvolution $[3,7,9]$. The Fermi model was fitted in the time domain whilst the distributed parameter model was fitted in the Laplace domain in order to avoid discontinuities of the time step-function that can be present when fitting the distributed parameter model in the time domain $[9,21]$. We fitted the convolution of the Fermi function with the first-pass of the arterial input function, setting the end-point at the CA concentration minimum before the recirculation component begins (this range varies from patient to patient but is commonly in the range between 20-35 dynamic frames). We also fitted the convolution of the distributed parameter function with the entire CA concentration time course of the arterial input function (i.e. 50 dynamic frames per slice). To further investigate the behaviour of distributed parameter modeling in single and dual bolus analysis, we also fitted the convolution of the distributed parameter model with the first-pass of the arterial input function using the same number of time points as in Fermi modeling. All additional microvascular parameters were calculated using the relationships described in Table $2[4,9]$.

A haematocrit value of 0.45 was assumed in order to convert myocardial blood flow into plasma flow which was used to calculate permeability surface area product, extraction fraction, extravascular-extracellular space and volume of distribution. Both models were fitted using a constrained nonlinear optimization (fmincon) in Matlab [22]. Myocardial perfusion reserve (myocardial blood flow at stress/ myocardial blood flow at rest) was calculated for all healthy volunteer data. Consistent with previous cardiac perfusion studies, vessel territories in patients with hyperaemic myocardial blood flow values less than $2.5 \mathrm{~mL} / \mathrm{min} / \mathrm{mL}$ of tissue were considered as regions with reduced myocardial blood flow $[5,6]$.

\section{Statistical analysis}

The $\mathrm{R}$ software was used for statistical analysis ( $\mathrm{R}$ Foundation for statistical computing, Vienna, Austria). Identification of any systematic bias between dual bolus and single bolus modeling estimates was performed using Bland Altman plots for both models. Statistical differences were investigated between Fermi and distributed parameter modeling, between distributed parameter and first-pass distributed parameter modeling, between stress and rest modeling values as well as between dual and single bolus analysis by implementing a paired $t$-test. A Welch two sample $t$-test was used to investigate statistical differences in myocardial blood flow values between the different groups (Groups 1-3) classified at the time of invasive coronary angiography.

Homogeneity of variances was verified using a Fisher's F-test. Comparison of mean myocardial blood flow and physiological parameters estimates in vessel territories of patients versus overall mean values in healthy volunteers was investigated using one sample $t$-test. Statistical significance was defined as two-sided $\mathrm{P}$ value $<0.05$.

\section{Results}

The distributed parameter model was fitted in 8 healthy volunteers and 5 patients with coronary artery disease. Example images are shown in Figure 1. We generated 416 CA concentration-time curves in 13 subjects (16 myocardial segments per subject both at stress and rest). Distributed parameter model fits were successful in 398 CA concentration-time curves and non-convergent in 7 myocardial segments of one volunteer at stress, in 5 myocardial segments of one volunteer at rest and in 6 segments of one patient at stress. The Fermi model successfully fitted all CA concentration-time courses.

\section{Comparison of Fermi and distributed parameter models in healthy volunteers}

We initially fitted the Fermi and distributed parameter models to CA concentration-time curves for our healthy volunteer population using arterial input functions derived from the main bolus data. Examples of Fermi and distributed parameter model fits at rest and stress are presented in Figure 2. Examples of pre-bolus and main bolus arterial input functions are shown in Figure 3. Fermi-derived myocardial blood flow values were higher than distributed parameter-derived myocardial blood flow values for both stress and rest $(\mathrm{P}=0.0005$ and $\mathrm{P}=0.007$ respectively, Table 3).

Table 1 Model equations

\begin{tabular}{llll}
\hline Model & Fitted parameters & Fitting domain & Tissue impulse response $\boldsymbol{R}$ \\
\hline Distributed parameter & Myocardial blood flow, $T_{,} T_{c}, T_{e}$ & Laplace & $R(s)=\frac{1-\exp \left[-s \cdot\left(T+s \cdot T_{c} \cdot T_{e}\right) /\left(1+s \cdot T_{e}\right)\right]}{s}$ \\
Fermi & Myocardial blood flow, $T_{0}, k$ & Time & $R(t)=\frac{1}{\exp \left[\left(t-T_{0}\right) \cdot k+1+1\right.}$
\end{tabular}

Fitted parameters for distributed parameter: myocardial blood flow, $\mathrm{T}$ is mean overall transit time, $\mathrm{T}_{\mathrm{c}}$ is mean capillary transit time, $\mathrm{T}_{\mathrm{e}}$ is mean interstitial (i.e. extravascular-extracellular) transit time. Where $s=i \cdot 2 \cdot \pi \cdot f$ and $f$ is the frequency variable in the Fourier transformed data. Fitted parameters for Fermi: myocardial blood flow, $\tau_{0}$ characterized the width of the shoulder of the Fermi function and k determined the decay rate of $\mathrm{R}(\mathrm{t})$ due to contrast agent wash-out. $t$ is the time variable. 
Table 2 Microvascular characteristics

\begin{tabular}{ll}
\hline Microvascular characteristics & Equation \\
\hline$v_{b}$ & $v_{b}=M B F \cdot T_{c}$ \\
$v_{e}$ & $v_{e}=M B F \cdot\left(T-T_{c}\right)$ \\
$v_{d}$ & $v_{d}=M B F \cdot T$ \\
$P S$ & $P S=\frac{M B F \cdot\left(T-T_{c}\right)}{T e}$ \\
$E$ & $E=1-\exp \left(-\frac{P S}{M P F}\right)$ \\
$M P F$ & $M P F=M B F \cdot(1-h c t)$
\end{tabular}

Microvascular characteristics were calculated by incorporating the fitted parameters of the distributed parameter model into the following relationships (see reference [4]). Myocardial plasma flow (MPF) was used to calculate extravascular-extracellular space $\left(v_{\mathrm{e}}\right)$, distribution volume $\left(\mathrm{v}_{\mathrm{d}}\right)$, permeability surface area product (PS) and extraction fraction (E) and myocardial blood flow (MBF) to calculate intravascular space $\left(\mathrm{v}_{\mathrm{b}}\right)$. Hematocrit: hct.

We subsequently fitted the Fermi and distributed parameter models for our healthy volunteer population, using scaled arterial input functions from their prebolus data. Fermi-derived myocardial blood flow values were again higher than distributed parameter-derived myocardial blood flow values for both stress and rest $(\mathrm{P}=0.03$ and $\mathrm{P}=0.003$ respectively, Table 3$)$.

Mean distributed parameter model-derived myocardial blood flow at stress was not different in dual bolus compared to single bolus analysis $(\mathrm{P}=0.22)$ whilst mean Fermi model-derived myocardial blood flow at stress was higher in single bolus versus dual bolus analysis ( $\mathrm{P}=0.00003$, Table 3).

Systematic bias of the above comparisons was investigated using Bland Altman method. The average bias was computed as the blood flow values at stress determined in dual bolus minus the relative values determined in the single bolus analysis. For the Fermi model, the average bias was -1.00 with $95 \%$ confidence intervals $[-1.58$, -0.42] and for the distributed parameter model, the average bias value was -0.30 with $95 \%$ confidence intervals $[-1.61,0.94]$.
Mean Fermi and distributed parameter-derived myocardial blood flow at rest did not significantly change between single and dual bolus analysis ( $\mathrm{P}=0.07$ for both). The additional distributed parameter estimates were not significantly different in single bolus compared to dual bolus analysis (see values in Additional file 1).

Mean myocardial blood flow was higher during hyperaemia in all healthy volunteers for distributed parameter-dual bolus ( $\mathrm{P}=0.00001)$, Fermi-dual bolus $(\mathrm{P}=0.0000001)$, distributed parameter-single bolus $(\mathrm{P}=0.0000005)$, and Fermi-single bolus analysis $(\mathrm{P}<$ $0.0000001)$. Mean (SD) myocardial perfusion reserve values were: 2.59 (0.37) for distributed parameter-dual bolus, $2.42(0.30)$ for distributed parameter-single bolus, 2.51 (0.48) for Fermi-dual bolus and 2.96 (0.34) for Fermi-single bolus analysis.

To investigate the lack of dependence of the distributed parameter model to arterial input function saturation observed in single bolus data, we also performed first-pass distributed parameter modeling. There was no difference between distributed parameter and first pass distributed parameter myocardial blood flow values ( $\mathrm{P}=0.17$ in dual bolus, $\mathrm{P}=0.79$ in single bolus analysis, Table 3). No difference was observed in firstpass distributed parameter-derived myocardial blood flow values between single and dual bolus analysis, for both stress $(P=0.31)$ and rest $(P=0.16)$ (Table 3$)$.

\section{Distributed parameter and Fermi analysis in patients with coronary artery disease}

Invasive coronary angiography and fractional flow reserve identified 7 vessels with obstructive lesions (Group 3), 5 vessels with non-obstructive lesions (Group 2 ) and 3 vessels with no or minor coronary artery disease (Group 1).

Mean myocardial blood flow values were calculated in vessel territories of the three main coronary arteries for each patient using both models (Table 4, Figure $2 \mathrm{e}$ and $\mathrm{f}$ ).
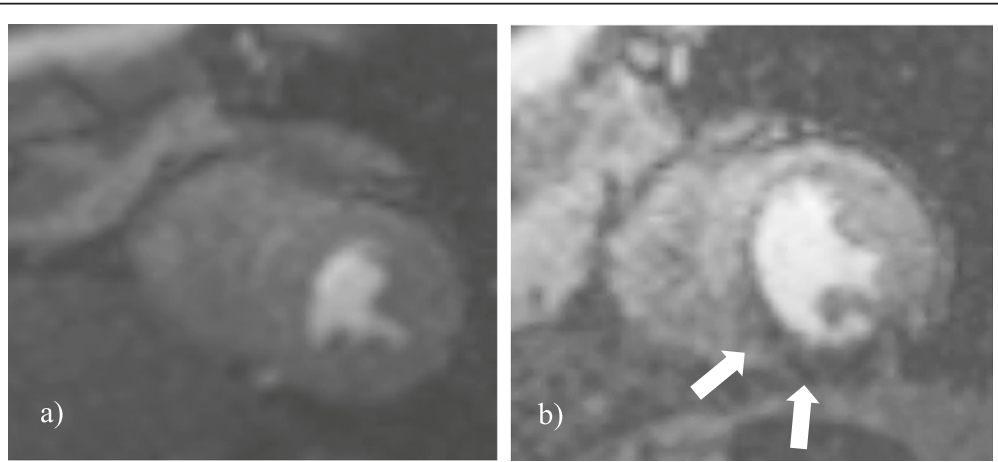

Figure 1 Mid-ventricular dynamic CMR perfusion images are shown. CMR perfusion image from a) a healthy volunteer and b) a patient with a perfusion abnormality in the infero-septal and inferior myocardial regions (white arrows). 


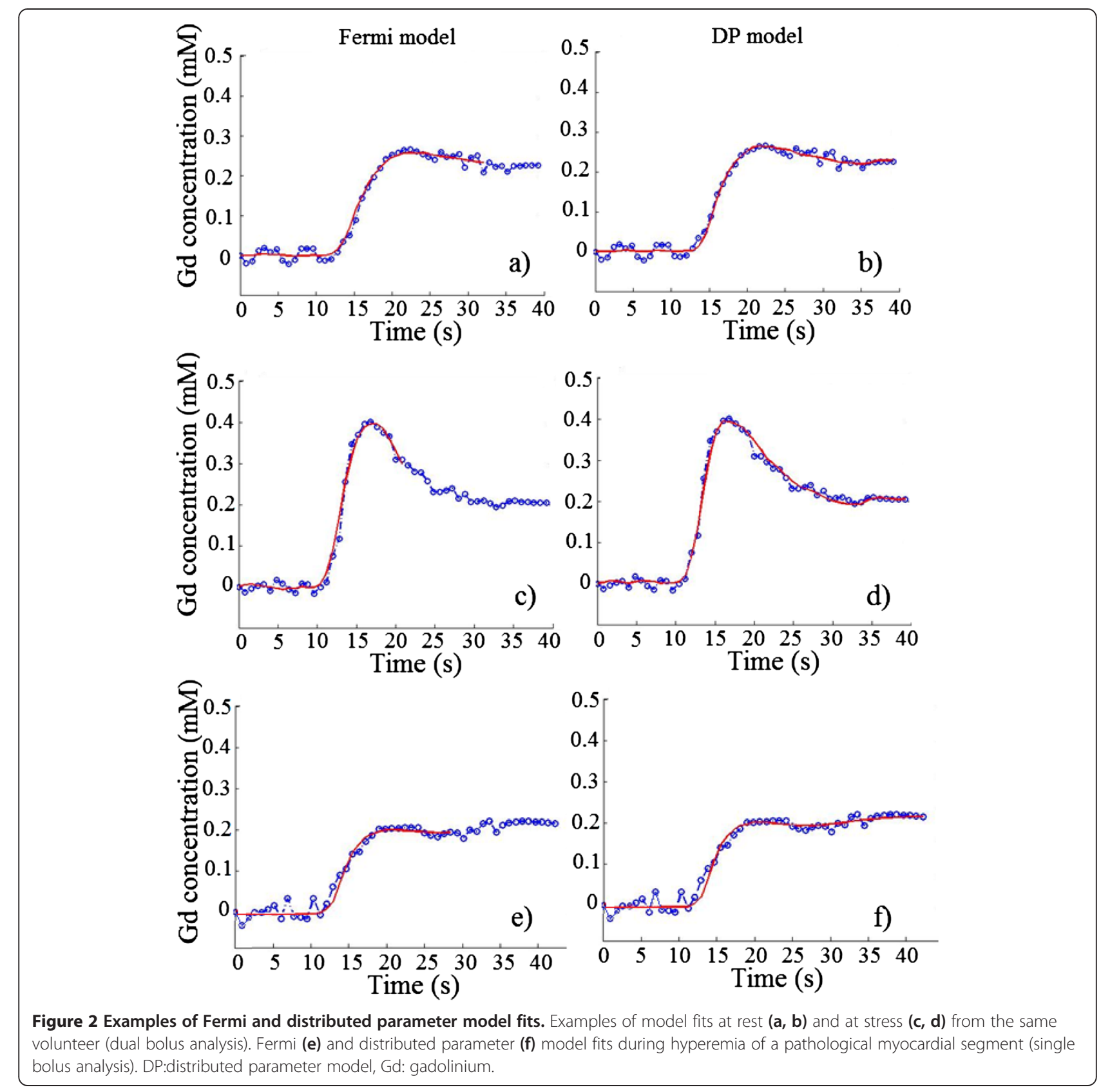

The Fermi and distributed parameter models correctly identified reduced myocardial blood flow in 6 and 7 of the 7 vessels in Group 3 respectively. In addition, the Fermi and distributed parameter models correctly identified reduced myocardial blood flow in 3 and 5 of the 5 vessels in Group 2 respectively. Both models estimated myocardial blood flow within normal range in Group 1. A difference was observed in myocardial blood flow at stress and in myocardial perfusion reserve between Group 1 versus Groups 2 and 3 for both models (Figure 4, Table 4).
Mean physiological parameter values were also calculated using distributed parameter modeling in all vessel territories for all patients (see Additional file 1).

\section{Discussion}

We have compared single and dual bolus estimates of myocardial perfusion in healthy volunteers using both Fermi and 2-region 1-barrier distributed parameter models. We demonstrate no difference in myocardial blood flow estimates using the distributed parameter model between single and dual bolus analysis. In 

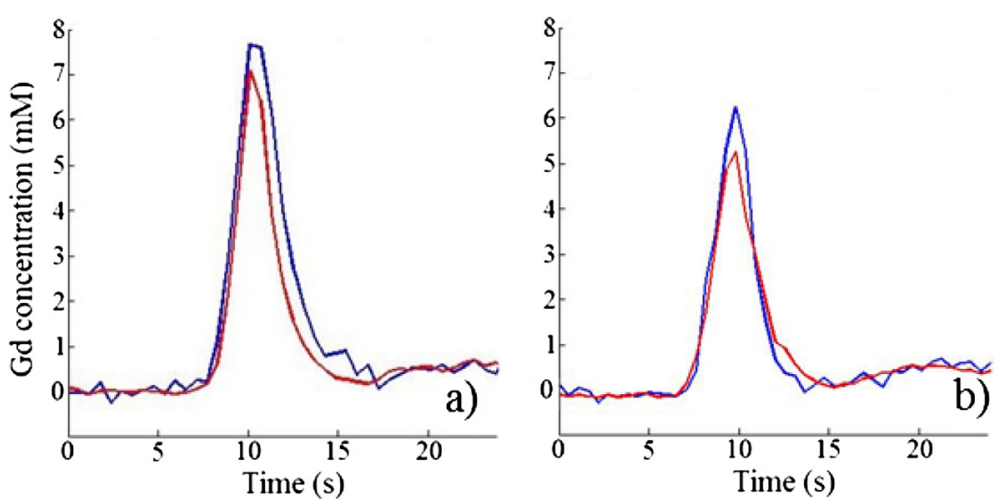

Figure 3 Scaled pre-bolus arterial input function versus standard arterial input function from the same examination. In volunteer 1 (a) and volunteer 2 (b) scaled pre-bolus (blue) arterial input function and main bolus arterial input function (red) are shown. Gd: gadolinium.

agreement with previous work, we demonstrate an increase in stress myocardial blood flow estimates with application of Fermi modeling using single bolus data analysis compared to dual bolus data analysis. For the first time, we have also successfully fitted the distributed parameter model in patients with coronary artery disease.

\section{Model comparison in healthy volunteers}

Using the distributed parameter model, we successfully fitted $96 \%$ of our data (398 in 416 CA concentration-time courses). Model comparison in 8 healthy volunteers suggested that single bolus analysis of the distributed parameter model shows no statistically significant difference compared to dual bolus analysis, indicating that this model may be less dependent on arterial input function saturation than the Fermi model. Furthermore, distributed parameter modeling using the first pass only, showed no statistically significant difference between single bolus and dual bolus analysis. This shows that the lack of dependence on single or dual bolus in the distributed parameter model using the full curve is not due to the increased number of time points used for fitting, compared to the first pass Fermi model. Dual bolus $[11,12,15,23]$ and dual sequence $[10,24]$ (which includes a low resolution dynamic acquisition of the left ventricle to eliminate arterial input function saturation), are the most widely suggested techniques to solve the issue of arterial input function saturation. However, both of these techniques involve increased complexity in image acquisition and data analysis that have led to ongoing debate regarding whether either method might replace standard single bolus protocols for CMR perfusion and myocardial blood flow quantification. Whilst single bolus protocols are prone to arterial input function saturation, they are still widely used in clinical imaging and are suitable for qualitative assessment of myocardial perfusion. Our analysis suggests that peak arterial input function saturation may not be such a dominant factor when quantifying myocardial blood flow in distributed parameter modeling compared with Fermi modeling.

Our calculated myocardial blood flow and microvascular characteristic parameter values generally agree with a previous study that was the first to introduce the two-region, one-barrier distributed parameter model in cardiac data [9]. Broadbent et al fitted a distributed parameter model in data acquired using a different protocol: short-axis view of the entire myocardial area across one mid-ventricular slice acquired in systole at $1.5 \mathrm{~T}$. We applied the distributed parameter model using a 16-segment heart model across three mid-ventricular slices acquired in diastole at 3T.

\section{The impact of contrast agent dose}

We validated the dependence of Fermi and distributed parameter modeling in the presence of arterial input function saturation in single bolus data, using a relatively low CA dose $(0.03 \mathrm{mmol} / \mathrm{kg})$ in our healthy volunteer cohort. The administration of the specific CA dose has

Table 3 Healthy volunteer mean (SD) myocardial blood flow values calculated using dual and single bolus analysis

\begin{tabular}{|c|c|c|c|c|c|c|}
\hline Modeling values/Method & $\begin{array}{l}\text { Fermi } \\
\text { Dual bolus }\end{array}$ & $\begin{array}{l}\text { Fermi } \\
\text { Single bolus }\end{array}$ & $\begin{array}{l}\text { DP } \\
\text { Dual bolus }\end{array}$ & $\begin{array}{l}\text { DP } \\
\text { Single bolus }\end{array}$ & $\begin{array}{l}\text { DP-First pass } \\
\text { Dual bolus }\end{array}$ & $\begin{array}{l}\text { DP-First pass } \\
\text { Single bolus }\end{array}$ \\
\hline Myocardial blood flow-Stress (mL/min/mL) & $3.57(0.59)^{*}$ & $4.57(0.62)^{*}$ & $3.16(0.71)$ & $3.45(0.48)$ & $3.39(0.56)$ & $3.47(0.50)$ \\
\hline Myocardial blood flow-Rest (mL/min/mL) & $1.48(0.40)$ & $1.57(0.33)$ & $1.23(0.26)$ & $1.46(0.29)$ & $1.18(0.26)$ & $1.34(0.31)$ \\
\hline
\end{tabular}

Statistical differences between single and dual bolus analysis are indicated with *. 
Table 4 Invasive coronary angiography/fractional flow reserve classification and mean myocardial blood flow (SD) at stress measured in $\mathrm{mL} / \mathrm{min} / \mathrm{mL}$ per vessel territories of the three main coronary arteries

\begin{tabular}{|c|c|c|c|c|c|c|}
\hline & & $\begin{array}{l}\text { Invasive coronary } \\
\text { angiography/Fractional } \\
\text { flow reserve }\end{array}$ & $\begin{array}{l}\text { Distributed } \\
\text { parameter-Myocardial } \\
\text { blood flow }\end{array}$ & $\begin{array}{l}\text { Fermi-Myocardial } \\
\text { blood flow }\end{array}$ & $\begin{array}{l}\text { Distributed } \\
\text { parameter-Myocardial } \\
\text { perfusion reserve }\end{array}$ & $\begin{array}{l}\text { Fermi-Myocardial } \\
\text { perfusion reserve }\end{array}$ \\
\hline \multirow[t]{3}{*}{ Patient 1} & LAD & 3 & $0.82(0.28)^{*}$ & $1.68(0.60)^{*}$ & $0.88(0.29)$ & $1.54(0.47)$ \\
\hline & LCX & 3 & $0.94(0.20)^{*}$ & $1.99(0.41)^{*}$ & $0.91(0.16)$ & $1.73(0.44)$ \\
\hline & RCA & 2 & $0.84(0.17)^{*}$ & $1.77(0.79)^{*}$ & $0.91(0.24)$ & $1.77(0.44)$ \\
\hline \multirow[t]{3}{*}{ Patient 2} & LAD & 2 & $1.99(0.30)^{*}$ & $3.37(0.49)$ & $1.68(0.37)$ & $2.15(0.49)$ \\
\hline & LCX & 2 & $1.98(0.27)^{*}$ & $2.61(0.41)$ & $1.26(0.30)$ & $1.87(0.80)$ \\
\hline & RCA & 3 & $1.27(0.27)^{*}$ & $1.80(0.81)^{*}$ & $0.86(0.30)$ & $1.08(0.33)$ \\
\hline \multirow[t]{3}{*}{ Patient 3} & LAD & 2 & $1.20(0.10)^{*}$ & $1.19(0.34)^{*}$ & $0.71(0.11)$ & $0.78(0.44)$ \\
\hline & LCX & 3 & $1.34(0.13)^{*}$ & $1.84(1.11)^{*}$ & $0.65(0.30)$ & $0.96(0.26)$ \\
\hline & RCA & 2 & $1.58(0.31)^{*}$ & $1.18(0.16)^{*}$ & $0.81(0.20)$ & $0.70(0.12)$ \\
\hline \multirow[t]{3}{*}{ Patient 4} & LAD & 3 & $1.99(0.31)^{*}$ & $3.02(0.64)$ & $1.21(0.31)$ & $1.22(0.23)$ \\
\hline & LCX & 3 & $1.61(0.73)^{*}$ & $1.98(0.58)^{*}$ & $0.90(0.35)$ & $1.05(0.34)$ \\
\hline & RCA & 3 & $0.75(0.29)^{*}$ & $1.00(0.44)^{*}$ & $0.58(0.23)$ & $0.65(0.24)$ \\
\hline \multirow[t]{3}{*}{ Patient 5} & LAD & 1 & $2.86(0.59)$ & $3.26(0.88)$ & $3.26(0.40)$ & $3.37(0.50)$ \\
\hline & LCX & 1 & $2.54(0.24)$ & $2.79(0.30)$ & $3.01(0.60)$ & $2.91(0.34)$ \\
\hline & RCA & 1 & $2.60(0.36)$ & $2.88(0.33)$ & $2.68(0.35)$ & $3.04(0.85)$ \\
\hline
\end{tabular}

LAD, LCX and RCA: left anterior descending, left circumflex and right coronary artery respectively. Vessels with reduced myocardial blood flow are indicated with *.

possibly caused limited arterial input function saturation at the peak of contrast enhancement $[3,10]$ (as shown in Figure 3), compared to higher CA doses. Our study demonstrates that Fermi modeling is still sensitive to any arterial input function saturation present in our single bolus data. In contrast, distributed parameter modeling is less dependent on any arterial input function saturation present in our data. Any increases in CA dose (at 3T), can increase the degree of arterial input function saturation in single bolus data of healthy volunteers, which would necessitate a de novo validation of distributed parameter modeling in single against dual bolus analysis.

\section{Distributed parameter and Fermi analysis in patients}

The distributed parameter model was capable of detecting reduced myocardial blood flow in patients with non-obstructive and obstructive coronary artery disease (Groups 2 and 3 respectively). Distributed parameter modeling correctly identified all 7 obstructive lesions and all 5 non-obstructive lesions. Fermi modeling correctly identified 6 out of 7 obstructive lesions and 3 out of 5 non-obstructive lesions. Both models showed decreased myocardial blood flow values as a function of luminal stenosis severity against invasive coronary angiography and fractional flow reserve classification (Figure 4).

\section{Study limitations}

The number of subjects included in this study is small. However, this is the first study demonstrating that a 1-barrier 2-region distributed parameter model approach may be less dependent on arterial input function saturation than Fermi modeling. Distributed parameter modeling needs to be applied in larger patient cohorts to further validate its diagnostic accuracy. We have not validated the behaviour of distributed parameter modeling in higher CA doses. To reduce patient discomfort during administration of adenosine, we did not implement a dual bolus stress-rest protocol in our patient cohort. As such, it was impossible to validate any systematic errors that may have contaminated our patient myocardial blood flow quantifications due to arterial input function saturation. To overcome this limitation and to complement our model comparison, we further assessed the ability of both models in detecting reduced myocardial blood flow in stenotic vessels versus current invasive gold standard methods. The CA dose used in patients was higher than in healthy volunteers to increase the signal-to-noise ratio due to an assumed reduction in blood flow in our patient cohort as compared to our healthy volunteer cohort. Although this higher dose in patients has possibly caused some myocardial blood flow overestimations in Fermi modeling (Table 4, in two epicardial vessels in patient 2 , and one epicardial vessel in patient 4), the distributed parameter modeling showed excellent accuracy in detecting reduced haemodynamics at stress in all stenotic vessels compared to our invasive gold standard. The vessels with non-obstructive disease (Group 2) were all from patients who also had one or two other vessels with obstructive disease (Group 3). 


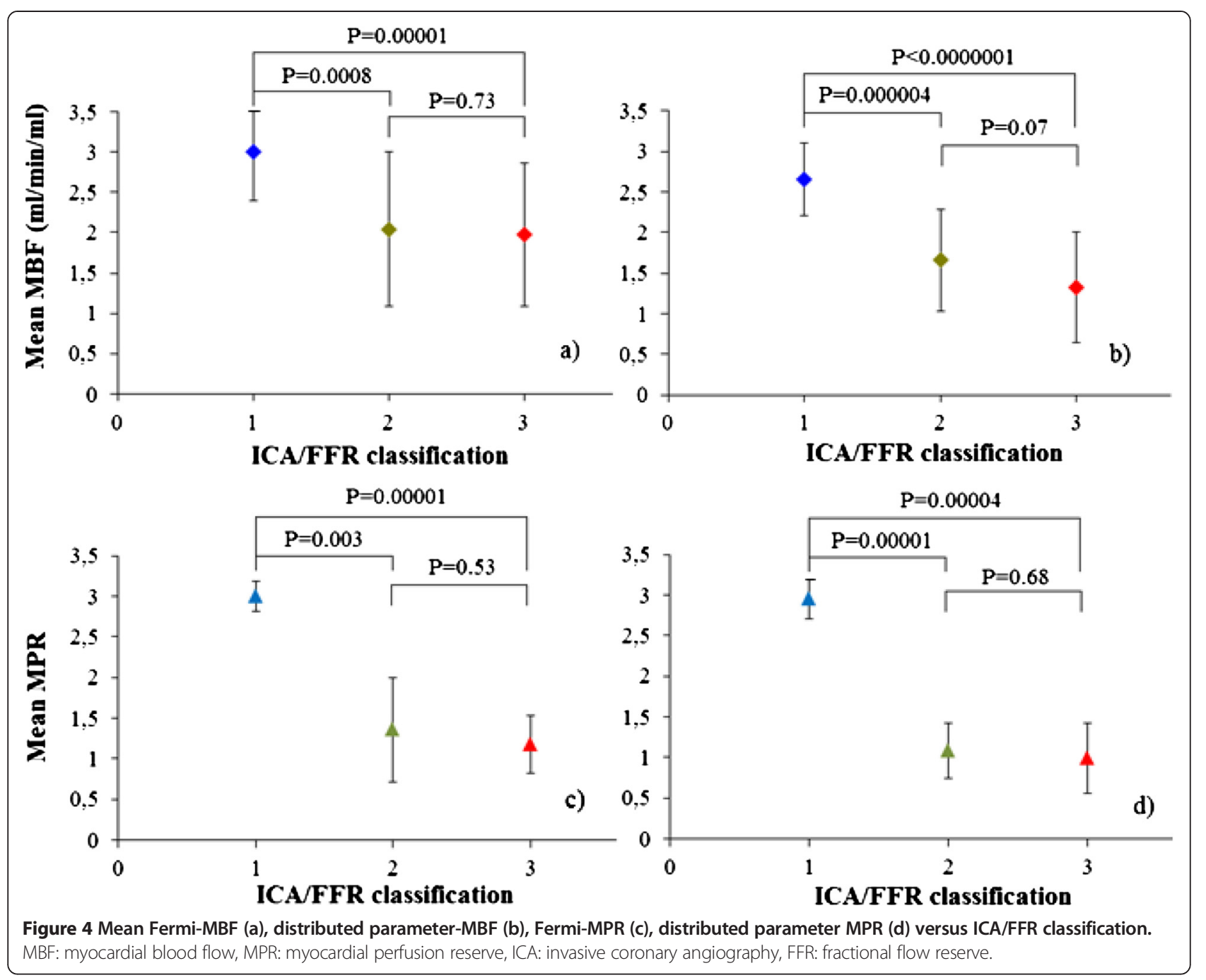

The coincidental effect of microvascular dysfunction could therefore explain the low myocardial blood flow measurements in Group 2.

\section{Conclusions}

We implemented a two-region, one-barrier distributed parameter model in healthy volunteers and patients with coronary artery disease. Distributed parameter-derived myocardial blood flow did not significantly change when a single bolus arterial input function was used compared to the dual bolus case. Fermi modelling of the same data demonstrated significant overestimations in myocardial blood flow in single bolus compared to dual bolus analysis. This suggests that the distributed parameter model might be less dependent on arterial input function saturation than Fermi modeling.

The distributed parameter model detected reduced myocardial blood flow in all 7 vessels with obstructive lesions and in all 5 vessels with non-obstructive lesions as determined by invasive coronary angiography and fractional flow reserve classification in a pilot cohort of 5 patients with coronary artery disease.

\section{Additional file}

Additional file 1: Mean microvascular characteristics (SD) estimates for healthy volunteers and for all 3 invasive coronary angiography/ fractional flow reserve Groups.

\section{Competing interests}

The authors declare that they have no competing interests.

\section{Authors' contributions}

GP: Author of the manuscript. Developed the hypothesis and conceptualized the objectives of the manuscript. Participated in the design of the single and dual bolus acquisition protocols, prepared the data analysis software and performed quantitative and statistical analysis. MW: Recruited all patients, participated in the preparation of healthy volunteers and patients before the magnetic resonance (MR) imaging and supervised the scans. LK: Supported the design of the image analysis software and helped in the design of the dual bolus protocol. SA: Prepared the healthy volunteers before the MR scanning and supervised the scans. MD: Prepared the healthy volunteers before the MR scanning and supervised the scans. SM: Performed qualitative assessment of the MR images and reported incidental findings and/or 
myocardial infarction. MC: Supported the design of the image analysis software. CG: Supported the design of the image analysis software3. TM: Supported the design of the image analysis software. DN: Revised the manuscript. Gave final approval to be published and approved the validity of the research questions and scientific approaches. Planned the study, obtained funding. SS: Revised the manuscript. Gave final approval to be published and approved the validity of the research questions, methodology and scientific approaches. Planned the study, obtained funding. All authors read and approved the final manuscript.

\section{Acknowledgements}

This work was made possible through funding and continued support from the British Heart Foundation. We would also like to thank the radiographers of the Clinical Research Imaging Centre for their help in image acquisition and input to the protocol.

\section{Funding}

British Heart Foundation (RE/08/001/23904).

\section{Author details}

${ }^{1}$ Clinical Research Imaging Centre, University of Edinburgh, Edinburgh, UK. ${ }^{2}$ Centre for Cardiovascular Science, University of Edinburgh, Edinburgh, UK. ${ }^{3}$ Christie Medical Physics and Engineering, The Christie NHS FT, Manchester, UK.

Received: 23 May 2014 Accepted: 22 January 2015

Published online: 17 February 2015

\section{References}

1. Hendel RC, Patel MR, Kramer CM, Poon M, Carr JC, Gerstad NA, et al. ACCF/ ACR/SCCT/SCMR/ASNC/NASCI/SCAI/SIR 2006 appropriateness criteria for cardiac computed tomography and cardiac magnetic resonance imaging. J Am Coll Cardiol. 2006:48:1475-97.

2. Jaarsma C, Leiner T, Bekkers SC, Crijns HJ, Wildberger JE, Nagel E, et al. Diagnostic performance of noninvasive myocardial perfusion imaging using single-photon emission computed tomography, cardiac magnetic resonance, and positron emission tomography imaging for the detection of obstructive coronary artery disease. J Am Coll Cardiol. 2012;59:1719-28.

3. Jerosch-Herold M. Quantification of myocardial perfusion by cardiovascular magnetic resonance. J Cardiov Magn Reson. 2010;12:57.

4. Sourbron SP, Buckley DL. Tracer kinetic modelling in MRl: estimating perfusion and capillary permeability. Phys in Med Biol. 2012;57:R1-33.

5. Nesterov SV, Han C, Mäki M, Kajander S, Naum AG, Helenius H, et al. Myocardial perfusion quantitation with 150-labelled water PET: high reproducibility of the new cardiac analysis software (Carimas). Eur J Nucl Med Mol Imaging. 2009;36:1594-602.

6. Kaufmann PA, Camici PG. Myocardial blood flow measurement by PET: technical aspects and clinical applications. J Nucl Med. 2005;46:75-88.

7. Biglands J, Magee D, Boyle R, Larghat A, Plein S, Radjenović A. Evaluation of the effect of myocardial segmentation errors on myocardial blood flow estimates from DCE-MRI. Phys in Med Biol. 2011;56(8):2423-43.

8. Jerosch-Herold M, Wilke N, Stillman AE. Magnetic resonance quantification of the myocardial perfusion reserve with a Fermi function model for constrained deconvolution. Med Phys. 1998;25:73-84.

9. Broadbent DA, Biglands JD, Larghat A, Sourbron SP, Radjenovic A, Greenwood JP, et al. Myocardial blood flow at rest and stress measured with dynamic contrast-enhanced MRl: Comparison of a distributed parameter model with a Fermi function model. Magn Reson Med. 2013;70:1591-7.

10. Gatehouse PD, Elkington AG, Ablitt NA, Yang G, Pennell DJ, Firmin DN. Accurate assessment of the arterial input function during high-dose myocardial perfusion cardiovascular magnetic resonance. J Magn Reson Im. 2004;20:39-45

11. Christian TF, Rettmann DW, Aletras AH, Liao SL, Taylor JL, Balaban RS, et al. Absolute myocardial perfusion in canines measured by using dual-bolus first-pass MR imaging. Radiology. 2004;232:677-84.

12. Utz W, Greiser A, Niendorf T, Diet R, Schulz-Menger J. Single- or dual-bolus approach for the assessment of myocardial perfusion reserve in quantitative MR perfusion imaging. Magn Reson Med. 2008;59:1373-7.

13. Messroghli DR, Greiser A, Fröhlich M, Dietz R, Schulz-Menger J. Optimization and validation of a fully-integrated pulse sequence for modified look-locker inversion-recovery (MOLLI) T1 mapping of the heart. J Magn Reson Im. 2007;26:1081-6.

14. Cerqueira MD. Standardized myocardial segmentation and nomenclature for tomographic imaging of the heart: a statement for healthcare professionals from the cardiac imaging committee of the council on clinical cardiology of the american heart association. Circulation. 2002;105:539-42.

15. Ishida M, Schuster A, Morton G, Chiribiri A, Hussain S, Paul M, et al. Development of a universal dual-bolus injection scheme for the quantitative assessment of myocardial perfusion cardiovascular magnetic resonance. J Cardiov Magn Reson. 2011;13:28.

16. Tonino PAL, Fearon WF, De Bruyne B, Oldroyd KG, Leesar MA, Ver Lee PN, et al. Angiographic versus functional severity of coronary artery stenoses in the FAME study. J Am Coll Cardiol. 2010;55:2816-21.

17. Tonino PAL, De Bruyne B, Pijls NHJ, Siebert U, Ikeno F, van't Veer M, et al. Fractional flow reserve versus angiography for guiding Percutaneous Coronary Intervention. N Engl J Med. 2009;360:213-24.

18. Kershaw LE, Hutchinson CE, Buckley DL. Benign prostatic hyperplasia: evaluation of T1, T2, and microvascular characteristics with T1-weighted dynamic contrast-enhanced MRI. J Magn Reson Im. 2009;29:641-8.

19. Larsson HBW, Fritz-Hansen T, Rostrup E, Søndergaard L, Ring P, Henriksen O. Myocardial perfusion modeling using MRI. Magn Reson Med. 1996;35:716-26.

20. Fritz-Hansen T, Rostrup E, Ring PB, Larsson HB. Quantification of gadolinium-DTPA concentrations for different inversion times using an IR-turbo flash pulse sequence: a study on optimizing multislice perfusion imaging. Magn Reson Med. 1998;16:893-9.

21. Garpebring A, Ostlund N, Karlsson M. A novel estimation method for physiological parameters in dynamic contrast-enhanced MRI: application of a distributed parameter model using Fourier-domain calculations. IEEE Trans Med Imaging. 2009;28:1375-83.

22. Byrd RH, Gilbert JC, Nocedal J. A trust region method based on interior point techniques for nonlinear programming. Math Program. 2000;89:149-85.

23. Hsu L-Y, Rhoads KL, Holly JE, Kellman P, Aletras AH, Arai AE. Quantitative myocardial perfusion analysis with a dual-bolus contrast-enhanced first-pass MRI technique in humans. Journal of magnetic resonance imaging. J Magn Reson Im. 2006:23:315-22.

24. Cernicanu A, Axel L. Theory-based signal calibration with single-point T1 measurements for first-pass quantitative perfusion MRI studies. Acad Radiol. 2006;13:686-93.

\section{Submit your next manuscript to BioMed Central and take full advantage of:}

- Convenient online submission

- Thorough peer review

- No space constraints or color figure charges

- Immediate publication on acceptance

- Inclusion in PubMed, CAS, Scopus and Google Scholar

- Research which is freely available for redistribution

Submit your manuscript at www.biomedcentral.com/submit
C Biomed Central 